\title{
On the tensor reduction of one-loop pentagons and hexagons
}

\author{
T. Diakonidis ${ }^{\mathrm{a}}$, J. Fleischer ${ }^{\mathrm{ab}}$, J. Gluza ${ }^{\mathrm{c}}$, K. Kajda ${ }^{\mathrm{c}}$, T. Riemann ${ }^{\mathrm{a}}$, J.B. Tausk ${ }^{\mathrm{a}}$ \\ a Deutsches Elektronen-Synchrotron, DESY, Platanenallee 6, 15738 Zeuthen, Germany \\ b Fakultät für Physik, Universität Bielefeld, Universitätsstr. 25, 33615 Bielefeld, Germany \\ ${ }^{c}$ Institute of Physics, University of Silesia, Uniwersytecka 4, 40-007 Katowice, Poland
}

We perform analytical reductions of one-loop tensor integrals with 5 and 6 legs to scalar master integrals. They are based on the use of recurrence relations connecting integrals in different space-time dimensions. The reductions are expressed in a compact form in terms of signed minors, and have been implemented in a mathematica package called hexagon.m. We present several numerical examples.

\section{Introduction}

Recent years have seen the emergence of first results for loop corrections to massive $2 \rightarrow 4$ scattering processes [1-3]. One of the challenges posed by such processes is the need to compute one-loop tensor integrals with up to 6 legs. In this contribution, we concentrate on integrals with 5 and 6 legs.

In 4-dimensional space-time, a linear relation must exist between the loop momentum and the external momenta of a pentagon integral. Similarly, if the external momenta of a hexagon are 4-dimensional, they must be linearly dependent. This enables one to reduce hexagons to pentagons, and pentagons to boxes [4-6]. These considerations have been extended to dimensionally regularized one-loop tensor integrals $[7,8]$. Generally, in the reduction of tensor integrals one encounters inverse Gram determinants, which can vanish at exceptional phase space points and be a source of instabilities in a numerical program. In the case of 4-dimensional pentagons, it is possible to avoid the leading inverse Gram determinant [9]. Other schemes for the reduction of multileg one-loop tensor integrals have been discussed in refs. [10-13].

Here, we use the methods of ref. [10]. Our present goal is to provide compact analytic formulas for the complete reduction of tensor pentagons and hexagons to scalar master integrals, which are free of leading inverse Gram determinants. We describe an implementation of these formulas in a mathematica package hexagon.m.

\section{Notations}

We consider one-loop, $N$-point tensor integrals of rank $R$ in $d$-dimensional space-time,

$J_{\mu_{1} \ldots \mu_{R}}^{(N)}\left(d ; \nu_{1}, \ldots, \nu_{N}\right)=\int \frac{d^{d} k}{i \pi^{d / 2}} \frac{k_{\mu_{1}} \ldots k_{\mu_{R}}}{D_{1}^{\nu_{1}} \ldots D_{N}^{\nu_{N}}}(1)$

with propagator denominators ${ }^{1}$

$D_{j}=\left(k-q_{j}\right)^{2}-m_{j}^{2}+i \epsilon$.

Following Davydychev [14], we decompose these tensor integrals into a basis of symmetric tensors constructed from metric tensors $g$ and the momenta $q_{j}$

$$
\begin{gathered}
J_{\mu_{1} \ldots \mu_{R}}^{(N)}\left(d ; \nu_{1}, \ldots, \nu_{N}\right)=(-1)^{R} \sum_{\lambda, \kappa_{1}, \ldots, \kappa_{N}}\left(-\frac{1}{2}\right)^{\lambda} \\
\left\{[g]^{\lambda}\left[q_{1}\right]^{\kappa_{1}} \ldots\left[q_{N}\right]^{\kappa_{N}}\right\}_{\mu_{1} \ldots \mu_{R}}\left(\nu_{1}\right)_{\kappa_{1}} \ldots\left(\nu_{N}\right)_{\kappa_{N}} \\
J^{(N)}\left(d+2(R-\lambda) ; \nu_{1}+\kappa_{1}, \ldots, \nu_{N}+\kappa_{N}\right)
\end{gathered}
$$

where $(\nu)_{\kappa}=\frac{\Gamma(\nu+\kappa)}{\Gamma(\nu)}$ are Pochhammer symbols and the sum runs over non-negative integers such

\footnotetext{
${ }^{1}$ In order to be consistent with the conventions of ref. [10], we have changed the sign of $q_{j}$ as compared with the original definition of ref. [14].
} 
that $2 \lambda+\kappa_{1}+\ldots+\kappa_{N}=R$. The next step is to use recurrence relations to reduce the scalar coefficients $J^{(N)}$ appearing in the decomposition to a set of master integrals.

It is useful to introduce a notation for certain determinants that occur in the recurrence relations and their solutions. First, the determinant of an $(N+1) \times(N+1)$ matrix, known as the modified Cayley determinant [4],

()$_{N} \equiv\left|\begin{array}{ccccc}0 & 1 & 1 & \ldots & 1 \\ 1 & Y_{11} & Y_{12} & \ldots & Y_{1 N} \\ 1 & Y_{12} & Y_{22} & \ldots & Y_{2 N} \\ \vdots & \vdots & \vdots & \ddots & \vdots \\ 1 & Y_{1 N} & Y_{2 N} & \ldots & Y_{N N}\end{array}\right|$,

with coefficients

$Y_{i j}=-\left(q_{i}-q_{j}\right)^{2}+m_{i}^{2}+m_{j}^{2}, \quad(i, j=1 \ldots N)$.

Although the masses of the propagators appear in the coefficients $Y_{i j}$, the determinant ()$_{N}$ does not depend on them and it is actually proportional to the Gram determinant of the external momenta of the $N$-point function in eq. (1). All other determinants we need are signed minors of ()$_{N}$, constructed by deleting $m$ rows and $m$ columns from ()$_{N}$, and multiplying with a sign factor. They will be denoted by

$$
\begin{aligned}
& \left(\begin{array}{cccc}
j_{1} & j_{2} & \cdots & j_{m} \\
k_{1} & k_{2} & \cdots & k_{m}
\end{array}\right)_{N} \equiv(-1)^{\sum_{l}\left(j_{l}+k_{l}\right)} \\
& \operatorname{sgn}_{\{j\}} \operatorname{sgn}_{\{k\}} \mid \begin{array}{c}
\text { rows } j_{1} \cdots j_{m} \text { deleted } \\
\text { columns } k_{1} \cdots k_{m} \text { deleted }
\end{array}
\end{aligned}
$$

where $\operatorname{sgn}_{\{j\}}$ and $\operatorname{sgn}_{\{k\}}$ are the signs of permutations that sort the deleted rows $j_{1} \cdots j_{m}$ and columns $k_{1} \cdots k_{m}$ into ascending order.

Combining integration by parts identities with relations connecting integrals in different spacetime dimensions [15], one obtains the following basic recurrence relations [10]:

$$
\begin{gathered}
()_{N} \nu_{j} \mathbf{j}^{+} J^{(N)}(d+2)= \\
{\left[-\left(\begin{array}{l}
j \\
0
\end{array}\right)_{N}+\sum_{k=1}^{n}\left(\begin{array}{l}
j \\
k
\end{array}\right)_{N} \mathbf{k}^{-}\right] J^{(N)}(d),} \\
\left(d-\sum_{i=1}^{n} \nu_{i}+1\right)()_{N} J^{(N)}(d+2)=
\end{gathered}
$$

$$
\begin{gathered}
{ }\left[\left(\begin{array}{l}
0 \\
0
\end{array}\right)_{N}-\sum_{k=1}^{n}\left(\begin{array}{l}
0 \\
k
\end{array}\right)_{N} \mathbf{k}^{-}\right] J^{(N)}(d), \\
\left(\begin{array}{l}
0 \\
0
\end{array}\right)_{N} \nu_{j} \mathbf{j}^{+} J^{(N)}(d)=\sum_{k=1}^{n}\left(\begin{array}{l}
0 j \\
0 k
\end{array}\right)_{N} \\
\times\left[d-\sum_{i=1}^{n} \nu_{i}\left(\mathbf{k}^{-} \mathbf{i}^{+}+1\right)\right] J^{(N)}(d) .
\end{gathered}
$$

where the operator $\mathbf{j}^{ \pm}$acts by shifting the index $\nu_{j}$ by \pm 1 .

\section{Pentagons}

A detailed discussion of the second rank pentagon is given in Ref. [16]. In this section, we will consider a third rank tensor integral with indices $\nu_{1}=\ldots=\nu_{5}=1$, which we write as $I_{5}^{\mu \nu \lambda}$. We assume here and in the next section, that the loop momentum $k$ has been shifted in such a way that $q_{N}=0$. Applying eq. (3) gives integrals in space-time dimensions $d+4$ and $d+6$ and with increased indices. They are reduced back to the generic dimension $d=4-2 \epsilon$ by the recurrence relations in eqs. (7)-(8). This involves a division by a Gram determinant ()$_{N}$ at each step. The leading Gram determinant, ()$_{5}$, can be avoided if one is only interested in contractions of the tensor integral with 4-dimensional objects [9]. This is achieved by using the following decomposition of the metric tensor,

$g^{\mu \nu}=2 \sum_{i, j=1}^{N-1} \frac{\left(\begin{array}{l}i \\ j\end{array}\right)_{N}}{()_{N}} q_{i}^{\mu} q_{j}^{\nu}+g_{\perp}^{\mu \nu}$,

and dropping terms proportional to $g_{\perp}^{\mu \nu}$. After further simplifications we obtain:

$I_{5}^{\mu \nu \lambda}=\sum_{i, j, k=1}^{4} q_{i}^{\mu} q_{j}^{\nu} q_{k}^{\lambda} E_{i j k}+\sum_{k=1}^{4} g^{[\mu \nu} q_{k}^{\lambda]} E_{00 k}$

where

$g^{[\mu \nu} q_{k}^{\lambda]}=g^{\mu \nu} q_{k}^{\lambda}+g^{\mu \lambda} q_{k}^{\nu}+g^{\nu \lambda} q_{k}^{\mu}$,

with scalar coefficients defined by

$E_{i j k}=\sum_{s=1}^{5} S_{i j k}^{4, s} I_{4}^{s}$ 


$$
+\sum_{s, t=1}^{5} S_{i j k}^{3, s t} I_{3}^{s t}+\sum_{s, t, u=1}^{5} S_{i j k}^{2, s t u} I_{2}^{s t u},
$$

with

$$
\begin{aligned}
& S_{i j k}^{4, s}=\frac{1}{3\left(\begin{array}{l}
0 \\
0
\end{array}\right)_{5}\left(\begin{array}{l}
s \\
s
\end{array}\right)_{5}^{2}} \times \\
& \left\{-\left(\begin{array}{c}
0 s \\
0 k
\end{array}\right)_{5}\left[\left(\begin{array}{c}
0 s \\
i s
\end{array}\right)_{5}\left(\begin{array}{c}
0 s \\
j s
\end{array}\right)_{5}+\left(\begin{array}{c}
i s \\
j s
\end{array}\right)_{5}\left(\begin{array}{c}
0 s \\
0 s
\end{array}\right)_{5}\right]\right. \\
& \left.+\left(\begin{array}{l}
0 s \\
0 s
\end{array}\right)_{5}\left[\left(\begin{array}{l}
0 i \\
s k
\end{array}\right)_{5}\left(\begin{array}{l}
0 s \\
j s
\end{array}\right)_{5}+\left(\begin{array}{l}
0 j \\
s k
\end{array}\right)_{5}\left(\begin{array}{l}
0 s \\
i s
\end{array}\right)_{5}\right]\right\} \\
& +(i \leftrightarrow k)+(j \leftrightarrow k), \\
& S_{i j k}^{3, s t}=\frac{1}{3\left(\begin{array}{l}
0 \\
0
\end{array}\right)_{5}\left(\begin{array}{l}
s \\
s
\end{array}\right)_{5}^{2}}\left\{( \begin{array} { c } 
{ 0 s } \\
{ 0 k }
\end{array} ) _ { 5 } \left[\left(\begin{array}{l}
t s \\
i s
\end{array}\right)_{5}\left(\begin{array}{l}
0 s \\
j s
\end{array}\right)_{5}\right.\right. \\
& \left.+\left(\begin{array}{c}
i s \\
j s
\end{array}\right)_{5}\left(\begin{array}{l}
t s \\
0 s
\end{array}\right)_{5}+\frac{\left(\begin{array}{l}
s \\
s
\end{array}\right)_{5}\left(\begin{array}{l}
0 s t \\
i s t
\end{array}\right)_{5}}{\left(\begin{array}{l}
s t \\
s t
\end{array}\right)_{5}}\left(\begin{array}{c}
t s \\
j s
\end{array}\right)_{5}\right] \\
& -\left[\left(\begin{array}{c}
0 i \\
s k
\end{array}\right)_{5}\left(\begin{array}{c}
0 s \\
j s
\end{array}\right)_{5}+\left(\begin{array}{c}
0 j \\
s k
\end{array}\right)_{5}\left(\begin{array}{c}
0 s \\
i s
\end{array}\right)_{5}\right]\left(\begin{array}{c}
t s \\
0 s
\end{array}\right)_{5} \\
& -\left[\left(\begin{array}{c}
0 i \\
s k
\end{array}\right)_{5}\left(\begin{array}{c}
t s \\
j s
\end{array}\right)_{5}+\left(\begin{array}{c}
0 j \\
s k
\end{array}\right)_{5}\left(\begin{array}{c}
t s \\
i s
\end{array}\right)_{5}\right] \\
& \left.\times \frac{\left(\begin{array}{c}
s \\
s
\end{array}\right)_{5}\left(\begin{array}{c}
0 s t \\
0 s t
\end{array}\right)_{5}}{2\left(\begin{array}{c}
s t \\
s t
\end{array}\right)_{5}}\right\}+(i \leftrightarrow k)+(j \leftrightarrow k), \\
& S_{i j k}^{2, s t u}=-\frac{1}{3\left(\begin{array}{l}
0 \\
0
\end{array}\right)_{5}\left(\begin{array}{l}
s \\
s
\end{array}\right)_{5}\left(\begin{array}{l}
s t \\
s t
\end{array}\right)_{5}} \times \\
& \left\{\left(\begin{array}{l}
0 s \\
0 k
\end{array}\right)_{5}\left(\begin{array}{c}
t s \\
j s
\end{array}\right)_{5}\left(\begin{array}{l}
u s t \\
i s t
\end{array}\right)_{5}-\frac{1}{2}\left[\left(\begin{array}{l}
0 j \\
s k
\end{array}\right)_{5}\left(\begin{array}{l}
u s t \\
i s t
\end{array}\right)_{5}\right.\right. \\
& \left.\left.+\left(\begin{array}{c}
0 i \\
s k
\end{array}\right)_{5}\left(\begin{array}{c}
u s t \\
j s t
\end{array}\right)_{5}\right]\left(\begin{array}{c}
t s \\
0 s
\end{array}\right)_{5}\right\} \\
& +(i \leftrightarrow k)+(j \leftrightarrow k),
\end{aligned}
$$

and

$$
\begin{aligned}
& E_{00 j}=\frac{1}{6\left(\begin{array}{l}
0 \\
0
\end{array}\right)_{5}}\left\{-\sum_{s=1}^{5} \frac{1}{\left(\begin{array}{l}
s \\
s
\end{array}\right)_{5}^{2}}\right. \\
& \times\left[3\left(\begin{array}{l}
s \\
0
\end{array}\right)_{5}\left(\begin{array}{l}
0 s \\
j s
\end{array}\right)_{5}-\left(\begin{array}{l}
s \\
j
\end{array}\right)_{5}\left(\begin{array}{l}
0 s \\
0 s
\end{array}\right)_{5}\right]\left(\begin{array}{l}
0 s \\
0 s
\end{array}\right)_{5} I_{4}^{s} \\
& +\sum_{s, t=1}^{5} \frac{1}{\left(\begin{array}{l}
s \\
s
\end{array}\right)_{5}^{2}} \\
& \times\left[3\left(\begin{array}{l}
s \\
0
\end{array}\right)_{5}\left(\begin{array}{c}
0 s \\
j s
\end{array}\right)_{5}-\left(\begin{array}{l}
s \\
j
\end{array}\right)_{5} \frac{\left(\begin{array}{c}
t s \\
0 s
\end{array}\right)_{5}^{2}}{\left(\begin{array}{c}
s t \\
s t
\end{array}\right)_{5}}\right]\left(\begin{array}{c}
t s \\
0 s
\end{array}\right)_{5} I_{3}^{s t}
\end{aligned}
$$

$$
\left.-\sum_{s, t, u=1}^{5}\left(\begin{array}{l}
s \\
j
\end{array}\right)_{5} \frac{\left(\begin{array}{c}
u s t \\
0 s t
\end{array}\right)_{5}}{\left(\begin{array}{c}
s \\
s
\end{array}\right)_{5}\left(\begin{array}{l}
s t \\
s t
\end{array}\right)_{5}}\left(\begin{array}{c}
t s \\
0 s
\end{array}\right)_{5} I_{2}^{s t u}\right\} .
$$

The decomposition in eq. (11) is equivalent to the one found in ref. [9], where the coefficients $E_{i j k}$ and $E_{00 j}$ are expressed in terms of tensor 4-point functions. Here, instead, they are completely reduced to a basis of scalar master integrals consisting of boxes $I_{4}^{s}$, vertices $I_{3}^{s t}$, and 2-point functions $I_{2}^{\text {stu }}$ obtained by removing lines $s, s$ and $t$, or $s$, $t$ and $u$ from the pentagon.

\section{Hexagons}

If the external momenta of a hexagon are 4dimensional, their Gram determinant vanishes: ()$_{6}=0$, and a linear relation between the propagators $D_{j}$ exists:

$1=\sum_{j=1}^{6} \frac{\left(\begin{array}{l}0 \\ j\end{array}\right)_{6}}{\left(\begin{array}{l}0 \\ 0\end{array}\right)_{6}} D_{j}$.

With this relation, any hexagon integral can trivially be reduced to pentagons. For example, for the scalar hexagon, one obtains the well-known result [4]:

$I_{6}=\sum_{r=1}^{6} \frac{\left(\begin{array}{l}0 \\ r\end{array}\right)_{6}}{\left(\begin{array}{l}0 \\ 0\end{array}\right)_{6}} I_{5}^{r}$,

where the scalar pentagon $I_{5}^{r}$ on the right hand side is obtained by removing line $r$ from the hexagon $I_{6}$. In the same way, tensor hexagons of rank $R$ can be reduced to tensor pentagons of rank $R$. However, it was noticed in ref. [10] that a reduction directly to tensor pentagons of rank $R-1$ is also possible:

$$
I_{6}^{\mu_{1} \ldots \mu_{R}}=\sum_{r=1}^{6} v_{r}^{\mu_{1}} I_{5}^{\mu_{2} \ldots \mu_{R}, r},
$$

where

$v_{r}^{\mu} \equiv-\frac{1}{\left(\begin{array}{l}0 \\ 0\end{array}\right)_{6}} \sum_{i=1}^{5}\left(\begin{array}{l}0 i \\ 0 r\end{array}\right)_{6} q_{i}^{\mu}$.

A more general proof of this property was given in ref. [13]. By substituting our reduction formulas for tensor pentagons into eq. (20), we can immediately express tensor hexagons in terms of scalar master integrals. 


\section{Numerical results}

The method of reducing six and five point Feynman integrals presented in this paper was implemented in a MATHEMATICA package called hexagon.m [17]. It allows to reduce six point integrals. Additionally, as hexagons are connected with pentagons, the package also allows one to reduce pentagons. The present implementation includes:

- six point functions up to rank four

- five point functions up to rank three .

These tensor ranks are sufficient to get results for e.g. NNLO contributions to Bhabha scattering. The kinematics used in the package ${ }^{2}$ is presented in Fig. 1.

Before using hexagon.m, the package must be loaded in a MATHEMATICA environment by executing:

\section{$<<$ hexagon.m}

The package is able to output the full result for a six or five point tensor integral, a specific coefficient, or a list of all coefficients for a given rank. For a more detailed description, see Table 1. hexagon. $\mathrm{m}$ is able to generate both analytic and numerical results, depending on the user's input. It provides coefficients of Lorentz-covariant tensors, and works in a basis of $g^{\mu \nu}$ and internal momenta (chords) $q_{i}$,

$q_{0}=0, \quad q_{n}=\sum_{i=1}^{n} p_{i}$,

see also Fig. 1. In terms of these coefficients, the tensor decomposition of pentagons $E$ and hexagons $F$ reads:

$$
\begin{aligned}
E^{\mu} & =\sum_{i=1}^{4} q_{i}^{\mu} E_{i}, \\
E^{\mu \nu} & =\sum_{i, j=1}^{4} q_{i}^{\mu} q_{i}^{\nu} E_{i j}+g^{\mu \nu} E_{00},
\end{aligned}
$$

\footnotetext{
${ }^{2}$ The masses and momenta are numbered according to the conventions of LoopTools [18], which are slightly different from those used in the previous sections and in ref. [10].
}
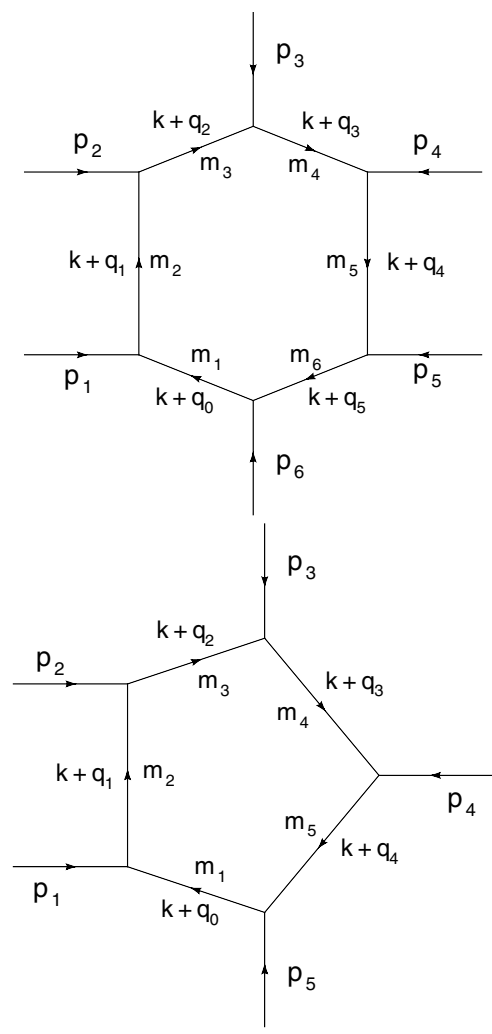

Figure 1. Momentum flow used in hexagon.m for six and five point diagrams.

$$
\begin{aligned}
E^{\mu \nu \lambda} & =\sum_{i, j, k=1}^{4} q_{i}^{\mu} q_{i}^{\nu} q_{k}^{\lambda} E_{i j k}+\sum_{i=1}^{4} g^{[\mu \nu} q_{i}^{\lambda]} E_{00 i}, \\
F^{\mu} & =\sum_{i=1}^{5} q_{i}^{\mu} F_{i}, \\
F^{\mu \nu} & =\sum_{i, j=1}^{5} q_{i}^{\mu} q_{i}^{\nu} F_{i j}, \\
F^{\mu \nu \lambda} & =\sum_{i, j, k=1}^{5} q_{i}^{\mu} q_{i}^{\nu} q_{k}^{\lambda} F_{i j k}+\sum_{i=1}^{5} g^{\mu \nu} q_{i}^{\lambda} F_{00 i}, \\
F^{\mu \nu \lambda \rho} & =\sum_{i, j, k, l=1}^{5} q_{i}^{\mu} q_{i}^{\nu} q_{k}^{\lambda} q_{l}^{\rho} F_{i j k l} \\
& +\sum_{i, j=1}^{5} q_{i}^{\mu} q_{j}^{[\nu} g^{\lambda \rho]} F_{00 i j} .
\end{aligned}
$$


Table 1

Functions used in the package.

\begin{tabular}{ll|ll}
\hline Six point functions & Five point functions \\
\hline RedF0 & scalar 6pt integral & RedE0 & scalar 5pt integral \\
RedF1 & vector 6pt integral & RedE1 & vector 5pt integral \\
RedF2 & rank two 6pt tensor integral & RedE2 & rank two 5pt tensor integral \\
RedF3 & rank three 6pt tensor integral & RedE3 & rank three 5pt tensor integral \\
RedF4 & rank four 6pt tensor integral & & \\
\hline RedFcoef & coefficient of given 6pt & RedEcoef & coefficient of given 5pt \\
RedFget & all coefficients of given 6pt & RedEget & all coefficients of given 5pt \\
\hline
\end{tabular}

The basic functions have the following arguments, here $s_{i j}=\left(p_{i}+p_{j}\right)^{2}, s_{i j k}=\left(p_{i}+p_{j}+p_{k}\right)^{2}$ :

$\operatorname{RedF0}\left[p_{1}^{2}, \ldots, p_{6}^{2}, s_{12}, s_{23}, s_{34}, s_{45}, s_{56}, s_{16}, s_{123}, s_{234}, s_{345}, m_{1}^{2}, \ldots, m_{6}^{2}\right]$

$\operatorname{RedE0}\left[p_{1}^{2}, \ldots, p_{5}^{2}, s_{12}, s_{23}, s_{34}, s_{45}, s_{15}, m_{1}^{2}, \ldots, m_{5}^{2}\right]$

For the purpose of checking the correctness of the reduction procedures implemented in the package, we have made both internal and external checks. Internal checks were used for tensor integrals, and consisted mainly in writing a scalar product of internal and external momenta in terms of lower rank tensor integrals, which had been checked before. External checks were made with use of: LoopTools [18] (five point integrals); AMBRE [19] with MB.m [20] (five point integrals); Sector Decomposition [21] (five and six point scalar integrals).

We present some of the cross-checks in Table 2. In all these checks, we use LoopTools to calculate the finite parts of the scalar four, three and two point functions which appear after the reduction by hexagon.m. We note that, in general, the functions defined directly in LoopTools may not be sufficient to cover the whole kinematic phase space obtained from reduction of six point functions. In such cases our reduction package must be supplemented with additional libraries.

In the first example in Table 2, we use AMBRE and MB.m to check the decomposition in the Euclidean kinematic region, including tensor structures. We show the result from hexagon.m for a contracted tensor of rank three. At the level typical for Monte Carlo calculations, it is in agreement with the result from Mellin-Barnes integration: 0.218885 .

The second example comes from ref. [22], (Table 2, region I). Here, we use the package [21] and show for the case of the scalar six point func- tion agreement with the calculation using the sector decomposition method (typically five digits accuracy). In the third example, we have extended the scalar case given in [22] (Table 1, region III). Also the tensorial results agree directly with LoopTools.

Finally, for six point tensor integrals, we have performed comparisons with a Fortran implementation of our reduction formulas by two of us (TD and BT), and also with an independent code by P. Uwer [23]. Some sample results, for the randomly chosen phase space point given in table 3 , are shown in table 4 .

\section{Summary}

We have described an analytical reduction of one-loop tensor integrals with 5 or 6 legs down to scalar master integrals, and shown explicitly the result for the tensor pentagon of rank three. Formulas for other cases and detailed derivations will be presented elsewhere. The reduction formulas have been implemented in a mathematica package hexagon.m which will be made publicly available.

\section{Acknowledgments}

We thank P. Uwer for discussions and for his kind assistance in cross checking our results for the tensor hexagons. Work supported by Sonderforschungsbereich/Transregio SFB/TRR 9 of DFG "Computergestützte Theoretische Teilchenphysik" and by the European Community's 
Table 2

Numerical cross-checks.

1) Comparison with AMBRE \& MB.m : $p_{1}^{\mu} p_{1}^{\nu} p_{1}^{\lambda} E_{\mu \nu \lambda}$

Point:

$p_{1}^{2}=p_{2}^{2}=p_{3}^{2}=p_{5}^{2}=1, p_{4}^{2}=0, m_{1}^{2}=m_{3}^{2}=0, m_{2}^{2}=m_{4}^{2}=m_{5}^{2}=1$,

$s_{12}=-3, s_{23}=-6, s_{34}=-5, s_{45}=-7, s_{15}=-2$

In: $\operatorname{RedE3}\left[p_{1}^{2}, \ldots, p_{5}^{2}, s_{12}, s_{23}, s_{34}, s_{45}, s_{15}, m_{1}^{2}, \ldots, m_{5}^{2}\right] / .\{\mathrm{D} 4->\mathrm{D} 0, \mathrm{C} 3->\mathrm{C} 0, \mathrm{~B} 2->\mathrm{B} 0\}$

Out : 0.218741

2) Comparison with Sector Decomposition: $F_{0}$

Point:

$p_{1}^{2}=p_{2}^{2}=p_{3}^{2}=p_{4}^{2}=p_{5}^{2}=p_{6}^{2}=-1, m_{1}^{2}=m_{2}^{2}=m_{3}^{2}=m_{4}^{2}=m_{5}^{2}=m_{6}^{2}=1$,

$s_{12}=s_{23}=s_{34}=s_{45}=s_{56}=s_{16}=s_{123}=s_{234}=-1, s_{345}=-5 / 2$

In: RedF0 $\left[p_{1}^{2}, \ldots, p_{6}^{2}, s_{12}, s_{23}, s_{34}, s_{45}, s_{56}, s_{16}, s_{123}, s_{234}, s_{345}, m_{1}^{2}, \ldots, m_{6}^{2}\right] / .\{\mathrm{D} 4->\mathrm{D} 0\}$

Out: 0.013526

3) Comparison with LoopTools : $E_{0}, E_{1}, E_{2}, E_{3}, E_{4}, E_{34}, E_{123}, E_{002}$

Point:

$p_{1}^{2}=p_{2}^{2}=0, p_{3}^{2}=p_{5}^{2}=49 / 256, p_{4}^{2}=9 / 100, m_{1}^{2}=m_{2}^{2}=m_{3}^{2}=49 / 256, m_{4}^{2}=m_{5}^{2}=81 / 1600$,

$s_{12}=4, s_{23}=-1 / 5, s_{34}=1 / 5, s_{45}=3 / 10, s_{15}=-1 / 2$

In: RedE0 $\left[p_{1}^{2}, \ldots, p_{5}^{2}, s_{12}, s_{23}, s_{34}, s_{45}, s_{15}, m_{1}^{2}, \ldots, m_{5}^{2}\right] / . \mathrm{D} 4->\mathrm{D} 0$

Out: $41.3403-45.9721 * I$

In: RedEget $\left[\right.$ rank1 $\left., p_{1}^{2}, \ldots, p_{5}^{2}, s_{12}, s_{23}, s_{34}, s_{45}, s_{15}, m_{1}^{2}, \ldots, m_{5}^{2}\right] /$. D4->D0

Out: ee1 $=-2.38605+5.27599 * I$, ee $2=-5.80875+0.597891 * I$,

ee3 $=-14.4931+20.8149 * I$, ee4 $=-11.3362+18.1593 * I$

In: RedEcoef $\left[\right.$ ee $\left.34, p_{1}^{2}, \ldots, p_{5}^{2}, s_{12}, s_{23}, s_{34}, s_{45}, s_{15}, m_{1}^{2}, \ldots, m_{5}^{2}\right] / .\{\mathrm{D} 4->\mathrm{D} 0, \mathrm{C} 3->\mathrm{C} 0\}$

Out: $7.1964+3.10115 * I$

In: RedEcoef $\left[\right.$ ee123 $\left., p_{1}^{2}, \ldots, p_{5}^{2}, s_{12}, s_{23}, s_{34}, s_{45}, s_{15}, m_{1}^{2}, \ldots, m_{5}^{2}\right] / .\{\mathrm{D} 4->\mathrm{D} 0, \mathrm{C} 3->\mathrm{C} 0, \mathrm{~B} 2->\mathrm{B} 0\}$

Out: $-0.149527-0.31059 * I$

In: RedEcoef $\left[\right.$ ee002 $\left., p_{1}^{2}, \ldots, p_{5}^{2}, s_{12}, s_{23}, s_{34}, s_{45}, s_{15}, m_{1}^{2}, \ldots, m_{5}^{2}\right] / .\{\mathrm{D} 4->\mathrm{D} 0, \mathrm{C} 3->\mathrm{C} 0, \mathrm{~B} 2->\mathrm{B} 0\}$

Out: $0.154517-0.387727 * I$

Marie-Curie Research Training Networks MRTNCT-2006-035505 "HEPTOOLS" and MRTN-CT2006-035482 "FLAVIAnet".

\section{REFERENCES}

1. A. Denner, S. Dittmaier, M. Roth and L. H. Wieders, Phys. Lett. B 612 (2005) 223 [arXiv:hep-ph/0502063]; A. Denner, S. Dittmaier, M. Roth and L. H. Wieders, Nucl. Phys. B 724 (2005) 247 [arXiv:hep$\mathrm{ph} / 0505042]$.

2. F. Boudjema et al., Proceedings of $L C W S$ 2005, Stanford, California, 18-22 Mar 2005, 0601 [arXiv:hep-ph/0510184].

3. S. Pozzorini, talk at this conference; A. Bredenstein, A. Denner, S. Dittmaier and S. Poz- zorini, arXiv:0807.1248 [hep-ph].

4. D. B. Melrose, Nuovo Cim. 40 (1965) 181.

5. W. L. van Neerven and J. A. M. Vermaseren, Phys. Lett. B 137 (1984) 241; W. L. van Neerven and J. A. M. Vermaseren, Phys. Lett. B 142 (1984) 80.

6. G. J. van Oldenborgh and J. A. M. Vermaseren, Z. Phys. C 46 (1990) 425.

7. Z. Bern, L. J. Dixon and D. A. Kosower, Phys. Lett. B 302 (1993) 299 [Erratum-ibid. B 318 (1993) 649] [arXiv:hep-ph/9212308]; Z. Bern, L. J. Dixon and D. A. Kosower, Nucl. Phys. B 412 (1994) 751 [arXiv:hepph/9306240].

8. T. Binoth, J. P. Guillet and G. Heinrich, Nucl. Phys. B 572 (2000) 361 [arXiv:hepph/9911342]. 
Table 3

Phase space point used in the tensor hexagons

$$
\begin{aligned}
& p_{1}=(0.21774554 E+03, \quad 0, \quad 0, \quad 0.21774554 E+03) \\
& p_{2}=(0.21774554 E+03, \quad 0, \quad 0, \quad-0.21774554 E+03) \\
& p_{3}=(-0.20369415 E+03, \quad-0.47579512 E+02, \quad 0.42126823 E+02, \quad 0.84097181 E+02) \\
& p_{4}=(-0.20907237 E+03, \quad 0.55215961 E+02, \quad-0.46692034 E+02, \quad-0.90010087 E+02) \\
& p_{5}=(-0.68463308 E+01, \quad 0.53063195 E+01, \quad 0.29698267 E+01, \quad-0.31456871 E+01) \\
& p_{6}=(-0.15878244 E+02, \quad-0.12942769 E+02, \quad 0.15953850 E+01, \quad 0.90585932 E+01) \\
& m_{1}=110.0, m_{2}=120.0, m_{3}=130.0, m_{4}=140.0, m_{5}=150.0, m_{6}=160.0
\end{aligned}
$$

\begin{tabular}{|c|c|c|c|}
\hline & & \multicolumn{2}{|c|}{ RESULTS } \\
\hline & & REAL & IM \\
\hline & & \multicolumn{2}{|c|}{$\overline{F_{0}}$} \\
\hline & & $-0.223393 \mathrm{E}-18$ & $-0.396728 \mathrm{E}-19$ \\
\hline$\mu$ & & \multicolumn{2}{|c|}{$\frac{1}{F^{\mu}}$} \\
\hline$\overline{0}$ & & $0.192487 \mathrm{E}-17$ & $0.972635 \mathrm{E}-17$ \\
\hline 1 & & $-0.363320 \mathrm{E}-17$ & $-0.11940 \mathrm{E}-17$ \\
\hline 2 & & $0.365514 \mathrm{E}-17$ & $0.106928 \mathrm{E}-17$ \\
\hline 3 & & $0.239793 \mathrm{E}-16$ & $0.341928 \mathrm{E}-17$ \\
\hline$\mu$ & $\nu$ & \multicolumn{2}{|c|}{$\frac{1}{F^{\mu \nu}}$} \\
\hline 0 & 0 & $0.599459 \mathrm{E}-14$ & $-0.114601 \mathrm{E}-14$ \\
\hline 0 & 1 & $0.323869 \mathrm{E}-15$ & $0.423754 \mathrm{E}-15$ \\
\hline 0 & 2 & $-0.294252 \mathrm{E}-15$ & $-0.375481 \mathrm{E}-15$ \\
\hline 0 & 3 & $-0.255450 \mathrm{E}-14$ & $-0.195640 \mathrm{E}-14$ \\
\hline 1 & 1 & $-0.164562 \mathrm{E}-14$ & $-0.993796 \mathrm{E}-16$ \\
\hline 1 & 2 & $0.920944 \mathrm{E}-16$ & $0.706487 \mathrm{E}-17$ \\
\hline 1 & 3 & $0.347694 \mathrm{E}-15$ & $-0.127190 \mathrm{E}-16$ \\
\hline 2 & 2 & $-0.163339 \mathrm{E}-14$ & $-0.994148 \mathrm{E}-16$ \\
\hline 2 & 3 & $-0.341773 \mathrm{E}-15$ & $0.818678 \mathrm{E}-17$ \\
\hline 3 & 3 & $-0.413909 \mathrm{E}-14$ & $0.670676 \mathrm{E}-15$ \\
\hline
\end{tabular}

Table 4

Results for scalar, vector, and 2nd-rank six point functions for the phase space point of Table 3 .

9. A. Denner and S. Dittmaier, Nucl. Phys. B 658 (2003) 175 [arXiv:hep-ph/0212259].

10. J. Fleischer, F. Jegerlehner and O. V. Tarasov, Nucl. Phys. B 566 (2000) 423 [arXiv:hep-ph/9907327].

11. G. Duplancic and B. Nizic, Eur. Phys. J. C 35 (2004) 105 [arXiv:hep-ph/0303184].

12. T. Binoth, J. P. Guillet, G. Heinrich, E. Pi- lon and C. Schubert, JHEP 0510 (2005) 015 [arXiv:hep-ph/0504267].

13. A. Denner and S. Dittmaier, Nucl. Phys. B 734 (2006) 62 [arXiv:hep-ph/0509141].

14. A. I. Davydychev, Phys. Lett. B 263 (1991) 107.

15. O. V. Tarasov, Phys. Rev. D 54 (1996) 6479 [arXiv:hep-th/9606018].

16. J. Fleischer, J. Gluza, K. Kajda and T. Riemann, Acta Phys. Polon. B 38 (2007) 3529 [arXiv:0710.5100 [hep-ph]].

17. K. Kajda, hexagon.m, available at http://www-zeuthen.desy.de/theory/ research/CAS.html

18. T. Hahn and M. Perez-Victoria, Comput. Phys. Commun. 118 (1999) 153 [arXiv:hepph/9807565]; T. Hahn and M. Rauch, Nucl. Phys. Proc. Suppl. 157 (2006) 236 [arXiv:hep-ph/0601248].

19. J. Gluza, K. Kajda and T. Riemann, Comput. Phys. Commun. 177 (2007) 879 [arXiv:0704.2423 [hep-ph]].

20. M. Czakon, Comput. Phys. Commun. 175 (2006) 559 [arXiv:hep-ph/0511200].

21. C. Bogner and S. Weinzierl, Comput. Phys. Commun. 178 (2008) 596 [arXiv:0709.4092 [hep-ph]].

22. T. Binoth, G. Heinrich and N. Kauer, Nucl. Phys. B 654 (2003) 277 [arXiv:hepph/0210023].

23. P. Uwer, private communications. 